\title{
Evaluation of methods for modeling transcription factor sequence specificity
}

\author{
Matthew T Weirauch ${ }^{1,2}$, Atina Cote ${ }^{1}$, Raquel Norel ${ }^{3}$, Matti Annala ${ }^{4}$, Yue Zhao ${ }^{5}$, Todd R Riley ${ }^{6}$, Julio Saez-Rodriguez ${ }^{7}$, \\ Thomas Cokelaer ${ }^{7}$, Anastasia Vedenko ${ }^{8}$, Shaheynoor Talukder ${ }^{1}$, DREAM5 Consortium ${ }^{9}$, Harmen J Bussemaker ${ }^{6}$, \\ Quaid D Morris ${ }^{1,10}$, Martha L Bulyk ${ }^{8,11,12}$, Gustavo Stolovitzky ${ }^{3}$, Timothy R Hughes ${ }^{1,10}$
}

\begin{abstract}
Genomic analyses often involve scanning for potential transcription factor (TF) binding sites using models of the sequence specificity of DNA binding proteins. Many approaches have been developed to model and learn a protein's DNA-binding specificity, but these methods have not been systematically compared. Here we applied 26 such approaches to in vitro protein binding microarray data for 66 mouse TFs belonging to various families. For nine TFs, we also scored the resulting motif models on in vivo data, and found that the best in vitro-derived motifs performed similarly to motifs derived from the in vivo data. Our results indicate that simple models based on mononucleotide position weight matrices trained by the best methods perform similarly to more complex models for most TFs examined, but fall short in specific cases ( $<10 \%$ of the TFs examined here). In addition, the best-performing motifs typically have relatively low information content, consistent with widespread degeneracy in eukaryotic TF sequence preferences.
\end{abstract}

Accurate modeling of the sequence specificities of TFs is of central importance for understanding the function and evolution of genomes. Ideally, sequence specificity models should predict the relative affinity (or dissociation constant) for different individual sequences and/or the probability of occupancy at any position in the genome. The major paradigm in modeling TF sequence specificity is the position weight matrix (PWM) model ${ }^{1-3}$. PWMs represent the DNA sequence preference of a TF as an $N$ by $B$ matrix, where $N$ is the length of the site bound by the TF, and $B$ is the number of possible nucleotide bases (that is, A, C, G or T). Each position provides a score for each nucleotide, representing the relative preference for the given base. PWM models provide an intuitive representation of the sequence preferences of a TF, including the exact position where it would bind the DNA, and involve relatively few parameters. However, recent studies suggest that shortcomings of PWMs, including their inability to model variable-width gaps, capture dependencies between the residues in the binding site or account for the fact that TFs can have more than one DNA-binding interface, can make them inaccurate $^{4-9}$. Alternative models have been developed that extend the PWM model by considering the contribution of combinations of nucleotides, for example, dinucleotides or combinations of multiple motifs $4,6,7,10$. Another alternative, $k$-mer-based approaches 7,11 , assign a score to every possible sequence of length $k$, and hence make no assumptions about position dependence, variable gap lengths or multiple binding motifs. To our knowledge, the relative efficacies of these approaches have not been systematically compared.

A major difficulty in studying TF-DNA binding specificity and, therefore, in evaluating models for representing this specificity has been scarcity of data. The process of training and testing models benefits from a large number of unbiased data points. In the case of TF-DNA binding models, the required data are the relative preferences of a TF for a large number of individual sequences. Ideally, such data should be obtained in an in vitro setting, as many confounding factors can influence the binding of a transcription factor in vivo (e.g., chromatin state, TF concentration or interactions with cofactors). Methods for measuring in vitro binding specificity include (HT)-SELEX/SELEXseq $^{12-15}$, HiTS-FLIP ${ }^{8}$, mechanically induced trapping of molecular interactions (MITOMI) ${ }^{9,16}$, cognate site identifier ${ }^{17}$, bacterial onehybrid ${ }^{18}$ and protein binding microarrays $(\mathrm{PBMs})^{19}$.

PBMs have enjoyed widespread use owing to the ease, accessibility and relatively high information content of the assay. Raw PBM data consist of a score (that is, fluorescence signal intensity) representing the relative preference of a given TF to the sequence of each probe contained on the array. PBM data represent specificity (that is, how strongly a given TF binds to a given sequence, relative to all other sequences), as opposed to binding affinity (that is, how strongly a TF

\footnotetext{
${ }^{1}$ Banting and Best Department of Medical Research and Donnelly Centre, University of Toronto, Toronto, Ontario, Canada. ${ }^{2}$ Center for Autoimmune Genomics and Etiology (CAGE) and Divisions of Rheumatology and Biomedical Informatics, Cincinnati Children's Hospital Medical Center, Cincinnati, Ohio, USA. ${ }^{3}$ BM Computational Biology Center, Yorktown Heights, New York, New York, USA. ${ }^{4}$ Department of Signal Processing, Tampere University of Technology, Tampere, Finland. ${ }^{5}$ Department of Genetics, University of Pennsylvania, Philadelphia, Pennsylvania, USA. ${ }^{6}$ Department of Biological Sciences, Columbia University, and Center for Computational Biology and Bioinformatics, Columbia University Medical Center, New York, New York, USA. ${ }^{7}$ EMBL-EBI European Bioinformatics Institute, Cambridge, UK. ${ }^{8}$ Department of Medicine, Division of Genetics, Brigham and Women's Hospital and Harvard Medical School, Boston, Massachusetts, USA. ${ }^{2}$ Full lists of members and affiliations appear at the end of the paper. ${ }^{10}$ Department of Molecular Genetics, University of Toronto, Toronto, Ontario, Canada. 11Department of Pathology, Brigham and Women's Hospital and Harvard Medical School, Boston, Massachusetts, USA. ${ }^{12}$ Harvard-MIT Division of Health Sciences and Technology, Harvard Medical School, Boston, Massachusetts, USA. Correspondence should be addressed to T.R.H. (t.hughes@utoronto.ca).
} 
binds to a single sequence); specificity is the more important measure, because in vivo, the TF must be able to distinguish its functional sites from all accessible sequences in the genome ${ }^{20}$. A typical universal $\mathrm{PBM}$ is designed using a de Bruijn sequence, such that all possible 10 -mers and 32 copies of every nonpalindromic 8-mer are contained within $\sim 40,00060$-base probe sequences (each containing either 35 or 36 unique bases) on each array, offering an unbiased survey of TF sequence specificities ${ }^{19}$. Constructing arrays with different de Bruijn sequences, each capturing the sequence specificities of the same TF to entirely different sets of sequences, provides a means to test the relative performance of various algorithms for modeling and predicting TF sequence specificities, because models can be trained on one array and tested on the other ${ }^{7,19}$. Here we present an evaluation of 26 different algorithms for modeling the DNA sequence specificity of a diversity of TFs, using two PBM array designs for each TF.

\section{RESULTS}

\section{The DREAM5 challenge}

The Dialogue for Reverse Engineering Assessments and Methods (DREAM) is a series of annual reverse-engineering systems biology challenges ${ }^{21-23}$. The DREAM5 TF-DNA Motif Recognition Challenge formed the basis for the analyses presented here. The challenge used PBM data to test the ability of different algorithms to represent the sequence preferences of TFs (here, 'algorithm' refers to the combination of data preprocessing, TF sequence specificity model, training and scoring). Briefly, we generated PBM data measuring the DNA sequence preferences of 86 mouse TFs, taken from 15 diverse TF families (Supplementary Table 1). All TFs were assayed in duplicate on two arrays with independent de Bruijn sequences (denoted 'ME' and 'HK' after the initials of the designers). In the DREAM5 challenge, the sequences of both arrays were made known, but only a subset of the PBM data was provided to participants, and the teams submitted predictions on the array data held back. For 20 randomly chosen TFs, array intensity data were provided from both array types, in order for the participants to calibrate and test their algorithms. For $33 \mathrm{TF}$, intensity data were provided only from the ME type of array; data for the remaining 33 TFs were provided only for the HK type of array. Given the output of probe intensities of one PBM array type, the challenge consisted of predicting the probe intensities of the second array type for each of the 66 TFs.

The probe intensity predictions from each participant were then evaluated using five criteria (that is, scores) that assess the ability of an algorithm to either predict probe sequence intensities or assign high ranks to preferred 8-mer sequences. These criteria and a combined score that summarizes the performance of each algorithm are described in Supplementary Note 1. Briefly, the $k$-mer-based method of Team_D ${ }^{11}$ outperformed all other algorithms, with algorithms ranked two through five performing similarly to each other (Supplementary Table 2). Of note, the top five teams represent a wide range of sequence specificity models (Table 1), suggesting that the algorithm, its implementation and its scoring system might be of greater importance than the type of model used.

The DREAM5 outcome, along with feedback from participants and others, led us to revisit and investigate several aspects of the results. First, we wanted to revisit the evaluation criteria. Second, we wanted to account for the possibility that microarray data preprocessing might have an effect on the final performance of a model or algorithm, as it clearly did for Team_ $\mathrm{D}^{11}$. Third, we wanted to incorporate published algorithms that were not represented in the challenge, including three biophysical energy-based algorithms, BEEMLPBM $^{24,25}$, FeatureREDUCE (T.R.R. and H.J.B., unpublished data) and MatrixREDUCE 26 , as well as two statistical algorithms, RankMotif $++^{27}$ and Seed-and-Wobble ${ }^{19}$. We also wanted to examine the impact of dinucleotide-based PWM models and 'secondary motifs', which can model proteins with multiple modes of binding $\mathrm{DNA}^{7}$. Here, we include 15 published and unpublished algorithms, in addition to 11 algorithms submitted as part of the original challenge (Table 1). Fourth, we wished to examine whether the results we obtained for in vitro data were supported by in vivo analyses and alternative in vitro assays.

\section{Revised evaluation criteria}

We considered two general issues in revisiting the evaluation criteria. The first is that, ideally, a representation of DNA sequence preference (e.g., a PWM) should output a number that reflects relative preference to a given sequence ${ }^{20}$. Most of the algorithms we considered aim to do this. In such cases it is reasonable to score using Pearson correlation. We note, however, that other models are intended to discriminate bound from unbound sets of sequences, or to represent the best binding sequences. In addition, microarray data can be subject to noise and saturation effects. In such cases it is appropriate to ask whether highly bound sequences can be discriminated from unbound sequences, which can be measured by the area under the receiver operating characteristic (AUROC).

The second issue is whether scoring should be based on predicting the 35-mer probe intensities or predicting their transformed 8 -mer values (we refer to full probe sequences as 35 -mers, because each 60 -base probe sequence contains 35 unique bases). The original DREAM5 challenge included both. There are arguments for and against both $7,19,24$ and our comparisons to independent data did not support either as being superior overall (Supplementary Note 2). In addition, the 8 -mer values can be derived by different means; one previous study ${ }^{7}$ directly predicted values for the test 8 -mers with PWMs, whereas another ${ }^{24}$ first scored the test 35 -mer scores and then converted these to 8 -mer scores. We found that the latter approach ${ }^{24}$ results in dramatically improved correlations to the measured test 8-mer $Z$-scores (Supplementary Note 2), suggesting that previous conclusions regarding secondary motifs, which were derived using the former approach ${ }^{7}$, should be revisited. Using the latter procedure ${ }^{24}$, the correlations obtained for 8-mers and for 35-mers on the same array scale with each other almost perfectly, whether the 35-mers are scored with PWMs or with 8-mers (Supplementary Note 2). The only substantial difference we have observed between scoring 35-mers or 8 -mers is that secondary motifs appear to confer a slight advantage when scoring 8-mers, but not 35-mers.

In the evaluations below, we use two criteria that are based on prediction of 35-mer intensities (which was the original DREAM5 challenge), but acknowledge that the data may be noisy and semiquantitative: (i) Pearson correlation between predicted and actual probe intensities (in the linear domain), and (ii) the AUROC of the set of positive probes, where positive probes are defined as those with actual intensities $>4$ s.d. above the mean probe intensity for the given experiment (average of 350 probes per experiment, out of $\sim 40,000$ probes total) (Fig. 1). We calculate a normalized score in which the top-performing algorithm for the given evaluation criterion receives a 1, and all other algorithms receive scores proportional to the top algorithm. The final score for an algorithm is the average of its two normalized scores. We also report the Pearson correlation between measured and predicted 8-mer scores, and the AUROC of positive 8 -mers, where positive 8 -mers are defined as those with associated $E$-scores $>0.45$ in the actual experiment ${ }^{28}$, although these are not used to gauge the efficacy of algorithms or models. 


\section{Results of new evaluations}

In the revised evaluations, we used the 35-mer scores from the DREAM5 challenge directly for eight of the algorithms. For the top three algorithms in the initial DREAM5 challenge that take $<24$ CPU hours to run per experiment (originally ranked 1, 3 and 4), as well as the algorithms BEEML-PBM ${ }^{24}$, FeatureREDUCE (T.R.R. and H.J.B., unpublished data), RankMotif $++{ }^{27}$, Seed-and-Wobble ${ }^{19}$ and five simple algorithms we implemented to provide a baseline (PWM_align, PWM_ align_E, 8mer_max, 8mer_sum and 8mer_pos), we constructed a training data set from the combination of preprocessing steps that resulted in the best final score for the given algorithm (Supplementary Note 3). Algorithms that were not subjected to the preprocessing analysis may work better if given the same benefit. We scored all 26 algorithms across the panel of 66 mouse TFs used in the challenge (see Supplementary Table 3 for all evaluation scores of each algorithm on each TF).
The results of our revised evaluation scheme produced rankings similar to those of the DREAM5 challenge, with the algorithm of Team_D again performing best among the original challenge participants (Table 2). Final performance was robust to the choice of evaluation criteria (Supplementary Fig. 1). Overall, the highestscoring algorithm is FeatureREDUCE, which combines a dinucleotide model in a biophysical framework with a background $k$-mer model explicitly intended to capture PBM-specific biases. In general, $k$-mer- and dinucleotide-based algorithms scored highest, although some PWM-based algorithms produced competitive results. Overall, it is notable that the specific algorithm is still more important than the type of sequence specificity model used by the algorithm. For example, BEEML-PBM, a published PWM-based algorithm, receives a better final score than four $k$-mer-based algorithms. Furthermore, algorithms based on the same sequence-specificity model type

Table 1 Summary of evaluated algorithms

\begin{tabular}{|c|c|c|}
\hline Name (rank) & Model type & Description of algorithm \\
\hline Team_D (1) ${ }^{11}$ & k-mers & $\begin{array}{l}\text { Constructs a matrix indexing the presence of contiguous } k \text {-mers (size } 4-8 \text { ) on each probe. } \\
\text { Estimates an affinity vector by applying a conjugate gradient method, and uses it to predict } \\
\text { intensities }{ }^{11} \text {. }\end{array}$ \\
\hline Team_F (2)/Dispom ${ }^{41}$ & Markov model & $\begin{array}{l}\text { Constructs a probabilistic classifier based on foreground and background Markov models. Weighted } \\
\text { extension of the Dispom algorithm. }\end{array}$ \\
\hline Team_E (3) & $\mathrm{PWM}+\mathrm{HMMs}$ & $\begin{array}{l}\text { Trains PWMs using MEME42, retrains by Expectation-Maximization using a Hidden Markov Model }{ }^{43} \text {, } \\
\text { and combines it with a probe-specific bias using a linear model. }\end{array}$ \\
\hline Team_G (4) & $k$-mers & $\begin{array}{l}\text { Models probe affinities as a product of an occurrence matrix of motif sequences (contiguous or } \\
\text { gapped 6-mers) and a vector of unknown motif affinities. Estimates motif affinities using a multiple } \\
\text { linear model. }\end{array}$ \\
\hline Team_J $(5)^{44,45}$ & Dinucleotides & $\begin{array}{l}\text { Trains binding energy linear models with nearest-neighbor dinucleotide contributions, and combines } \\
\text { them with probe sequence-dependent bias under an information theory-based framework }{ }^{44,45} \text {. }\end{array}$ \\
\hline Team_I (6) / Amadeus ${ }^{46}$ & k-mers + PWM & $\begin{array}{l}\text { Identifies and scores } 20 \text { de novo PWM models using Amadeus }{ }^{46} \text {. Combines the PWM with } \\
\text { maximum probe sequence contiguous } 6 \text {-mer AUC scores, and performs linear regression against } \\
\text { the probe intensities. }\end{array}$ \\
\hline Team_C (7) & PWM + k-mers + Random forests & $\begin{array}{l}\text { Constructs blended predictions from random forests of contiguous k-mers (length } 4 \text { through } 6 \text { ) } \\
\text { and RankMotif }++^{27} \text { PWMs. }\end{array}$ \\
\hline Team_H (7) 10 & $k$-mers + dinucleotides & $\begin{array}{l}\text { Trains support vector regression models to directly learn the mapping from probe sequences (using } \\
\text { inexact matches to dinucleotide } k \text {-mers of length } 10 \text { to } 15 \text { ) to the measured binding intensity }{ }^{10} \text {. }\end{array}$ \\
\hline Team_A (10) & $k$-mers & $\begin{array}{l}\text { Uses top 1,000 and bottom } 250 \text { 8-mers for specific binding, and nucleotide triplet background } \\
\text { frequencies for nonspecific binding. Performs linear regression between these features and the } \\
\text { observed binding intensities using Lasso } 47 \text {. }\end{array}$ \\
\hline Team_K (11) & $k$-mers & $\begin{array}{l}\text { Identifies informative contiguous } k \text {-mers (length } 1 \text { to } 8 \text { ) using feature selection (allowing mis- } \\
\text { matches), learns their weights using regression against the probe intensities. }\end{array}$ \\
\hline Team_B (13) & PWM & $\begin{array}{l}\text { Uses top and bottom } 1,000 \text { probes as positive and negative sets for discriminative motif discovery } \\
\text { using eTFBS } 48 \text {. Uses PWM scores as features for constructing regression models. }\end{array}$ \\
\hline BEEML-PBM 24,25 & PWM or dinucleotides & $\begin{array}{l}\text { Obtains maximum likelihood estimates of parameters to a biophysical PWM }{ }^{24} \text { or dinucleotide } 25 \\
\text { model, including the TF's chemical potential, nonspecific binding affinity, and probe position-spe- } \\
\text { cific effects. }\end{array}$ \\
\hline FeatureREDUCE & PWM, dinucleotides and/or k-mers & $\begin{array}{l}\text { Combines a biophysical free energy model (PWM or dinucleotide) with a contiguous } k \text {-mer } \\
\text { background model (length } 4 \text { to } 8 \text { ) in a robust regression framework. Throughout, we use 'Feature- } \\
\text { REDUCE' to denote the combined dinucleotide and } k \text {-mer model, FeatureREDUCE_PWM to denote } \\
\text { the PWM-only model, and FeatureREDUCE_dinuc to denote the dinucleotide-only model. }\end{array}$ \\
\hline MatrixREDUCE26 & PWM & $\begin{array}{l}\text { Performs a least-squares fit to a statistical-mechanical PWM model to discover the relative } \\
\text { contributions to the free energy of binding for each nucleotide at each position }{ }^{26} \text {. }\end{array}$ \\
\hline RankMotif ++27 & PWM & $\begin{array}{l}\text { Trains PWMs by maximizing the likelihood of a set of binding preferences under a probabilistic } \\
\text { model of how sequence binding affinity translates into binary binding preference observations }{ }^{27} \text {. }\end{array}$ \\
\hline Seed-and-Wobble 19 & PWM & $\begin{array}{l}\text { Uses the } 8 \text {-mer with the highest } E \text {-score as a seed, and inspects all single-mismatch variants } \\
\text { (and positions flanking the seed sequence) to identify the relative contribution of each base at } \\
\text { each position to the binding specificity }{ }^{19} \text {. }\end{array}$ \\
\hline 8mer_max & $k$-mers & $\begin{array}{l}\text { Calculates the median probe score of all contiguous 8-mers. Prediction is the maximum 8-mer } \\
\text { score on each probe. }\end{array}$ \\
\hline 8mer_pos & $k$-mers & $\begin{array}{l}\text { Similar to 8mer_sum, but takes into account probe position effect in a manner similar to } \\
\text { BEEML-PBM. }\end{array}$ \\
\hline 8mer_sum & k-mers & $\begin{array}{l}\text { Calculates the median probe score of all contiguous 8-mers. Prediction is the sum of all 8-mer } \\
\text { scores on each probe. }\end{array}$ \\
\hline PWM_align & PWM & Aligns all contiguous 8-mers with $E$-score $>0.45$ to create a PWM. \\
\hline PWM_align_E & PWM & $\begin{array}{l}\text { Aligns all contiguous } 8 \text {-mers with } E \text {-score }>0.45 \text {, weighting each sequence by its } E \text {-score, to } \\
\text { create a PWM. }\end{array}$ \\
\hline
\end{tabular}

The type of sequence specificity model used by each algorithm is indicated, along with a brief description of the algorithm (more information about the algorithms can be found in Supplementary Note 9). The final rank in the original DREAM5 challenge is indicated in parenthesis after the algorithm's name, where applicable. 
a

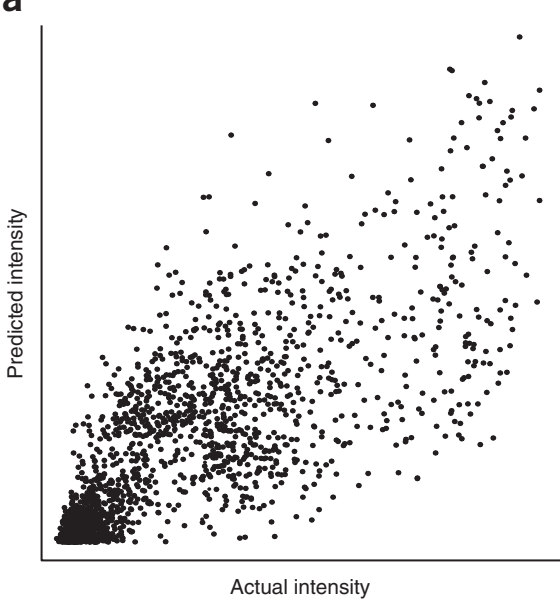

b

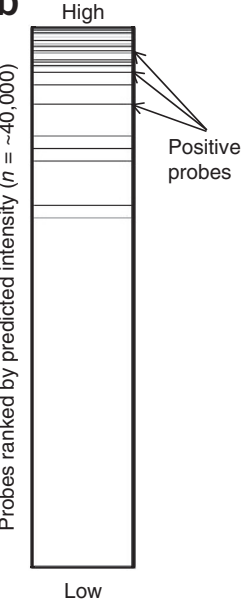

Figure 1 Evaluation criteria used in this study. For each TF, we scored an algorithm's probe intensity predictions using two evaluation criteria, which are illustrated here for TF_16 (Prdm11), using the predictions of BEEMLPBM on the raw array intensity data. (a) Pearson correlation between predicted and actual probe intensities across all $\sim 40,000$ probes. (b) AUROC of the set of positive probes. Positive probes (black lines) were defined as all probes on the test array with intensities $>4$ s.d. above the mean probe intensity for the given array.

(e.g., PWM, dinucleotides or $k$-mers) do not necessarily produce similar probe intensity predictions (Supplementary Fig. 2).

Algorithm performance varied substantially across the 66 TFs (Fig. 2a). The quality of the underlying experimental data, as opposed to inherent differences between TF families, appears to be the major factor in the overall ease of predicting probe intensities for a given TF (Fig. 2b and Supplementary Note 4). For example, TFs that were harder for most algorithms to model tended to have lower correlations between the 8-mer $Z$-scores of their training and test arrays, and fewer 8-mer $E$-scores $>0.45$ on their training arrays.

To further examine the relative performance of the $k$-mer, dinucleotide and PWM models, we compared the final scores produced by the single algorithm from each model category that performed best for each TF. On average, the best $k$-mer-based algorithm outperformed the best dinucleotide or PWM algorithm, but this is mainly because of large differences in a handful of specific TFs (Fig. 2b,c). Algorithms based on dinucleotides did substantially worse on these harder-to-model TFs, suggesting that they might be overfitting to array-specific noise. The best PWM-based algorithm performs as well as the best $k$-mer-based algorithm for the majority of TFs (Fig. 2c), with a median difference of only 0.014. PWM algorithms, in fact, did slightly better than $k$-mer-based algorithms for 18 TFs (Fig. 2c). However, of the five cases in which the final score for the best of one model type beats the best of the other type by $>0.10$, all but one favor $k$-mer algorithms (Fig. 2c). The majority of TFs for which the $k$-mer model performed better contained $\mathrm{C}_{2} \mathrm{H}_{2}$ zinc-finger arrays, which, depending on which $\mathrm{C}_{2} \mathrm{H}_{2}$ fingers are engaged, may have different binding modes; there is previous evidence for such phenomena both in vivo and in vitro ${ }^{7,29}$. However, some of these $\mathrm{C}_{2} \mathrm{H}_{2}$ zinc fingers present a challenge for all sequence-specificity models, perhaps owing to the small number of sequences they preferentially bind (Fig. 2 and Supplementary Note 4 ).

Despite the fact that more complicated algorithms produce higher scores, the results of these analyses suggest that the PWM model can accurately capture the sequence preferences for most TFs. Nevertheless, we observed a wide range in PWM-based algorithm performance across the 66 TFs (Fig. 2a). The fact that the two highestscoring PWM-based algorithms, Team_E and BEEML-PBM (Table 2), both model PBM-specific effects suggests that their high scores might not be solely due to superior PWMs. We carried out a series of analyses aimed at isolating the predictive ability of the PWMs produced by all of the PWM-based algorithms. Those produced by BEEML-PBM were the most accurate of all of the algorithms; the high performance of Team_E is due to its extensive modeling of PBM background effects and not to the quality of its PWMs (Supplementary Note 5). We also found this to be the case for predicting in vivo TF binding (see below).

\section{Analysis of dinucleotide matrices and secondary motifs}

Numerous studies have called into question the accuracy of the assumption inherent to the PWM model that bases are independent and many propose the use of dinucleotide dependencies to model TF binding. To quantify the relative accuracies of the dinucleotide and PWM models, we compared the performance of two of the top

Table 2 Final evaluation results

\begin{tabular}{|c|c|c|c|c|c|c|c|}
\hline Rank & Algorithm & Model & $\begin{array}{l}\text { Final } \\
\text { score }\end{array}$ & $\begin{array}{c}\text { Corr } \\
\text { (probes) }\end{array}$ & $\begin{array}{c}\text { AUROC-0.5 } \\
\text { (probes) }\end{array}$ & $\begin{array}{c}\text { Corr } \\
\text { (8-mers) }\end{array}$ & $\begin{array}{c}\text { AUROC-0.5 } \\
\text { (8-mers) }\end{array}$ \\
\hline 1 & FeatureREDUCE & Hybrid & 0.997 & 0.693 & 0.449 & 0.786 & 0.497 \\
\hline 2 & Team_D & $k$-mer & 0.984 & 0.691 & 0.438 & 0.820 & 0.496 \\
\hline 3 & Team_E & PWM & 0.952 & 0.696 & 0.406 & 0.761 & 0.447 \\
\hline 4 & Team_G & $k$-mer & 0.950 & 0.652 & 0.433 & 0.767 & 0.494 \\
\hline 5 & FeatureREDUCE_dinuc & Dinuc & 0.924 & 0.624 & 0.428 & 0.694 & 0.490 \\
\hline 6 & BEEML-PBM_dinuc & Dinuc & 0.919 & 0.623 & 0.424 & 0.738 & 0.488 \\
\hline 7 & Team_Fa & Other & 0.901 & 0.610 & 0.416 & 0.764 & 0.476 \\
\hline 8 & 8mer_pos & $k$-mer & 0.899 & 0.603 & 0.419 & 0.765 & 0.490 \\
\hline 9 & BEEML-PBM & PWM & 0.898 & 0.607 & 0.415 & 0.722 & 0.479 \\
\hline 10 & 8mer_sum & $k$-mer & 0.896 & 0.598 & 0.419 & 0.766 & 0.490 \\
\hline 11 & Team_Ja & Other & 0.895 & 0.611 & 0.410 & 0.740 & 0.465 \\
\hline 12 & FeatureREDUCE_PWM & PWM & 0.880 & 0.586 & 0.413 & 0.647 & 0.485 \\
\hline 13 & 8mer_max & $k$-mer & 0.846 & 0.541 & 0.411 & 0.688 & 0.494 \\
\hline 14 & Team_|a & Other & 0.813 & 0.581 & 0.356 & 0.683 & 0.439 \\
\hline 15 & BEEML-PBM_sec & 2 PWMs & 0.812 & 0.539 & 0.382 & 0.671 & 0.477 \\
\hline 16 & Team_ca & Other & 0.812 & 0.517 & 0.396 & 0.664 & 0.476 \\
\hline 17 & MatrixREDUCE & PWM & 0.791 & 0.526 & 0.371 & 0.669 & 0.455 \\
\hline 18 & FeatureREDUCE_sec & 2 PWMs & 0.790 & 0.508 & 0.382 & 0.610 & 0.482 \\
\hline 19 & Team_Aa & k-mer & 0.789 & 0.533 & 0.365 & 0.671 & 0.414 \\
\hline 20 & Team_H ${ }^{a}$ & Other & 0.778 & 0.468 & 0.397 & 0.625 & 0.491 \\
\hline 21 & PWM_align & PWM & 0.768 & 0.493 & 0.372 & 0.641 & 0.462 \\
\hline 22 & PWM_align_E & PWM & 0.757 & 0.511 & 0.351 & 0.666 & 0.468 \\
\hline 23 & Team_Ka & k-mer & 0.702 & 0.461 & 0.333 & 0.561 & 0.430 \\
\hline 24 & Seed-and-Wobble & PWM & 0.647 & 0.324 & 0.372 & 0.303 & 0.460 \\
\hline 25 & RankMotif++ & PWM & 0.582 & 0.275 & 0.346 & 0.408 & 0.460 \\
\hline 26 & Team_Ba & PWM & 0.509 & 0.266 & 0.286 & 0.354 & 0.393 \\
\hline
\end{tabular}

Algorithms are ranked by their final score, which is a combination of the evaluation criteria indicated in the columns labeled "Corr (probes)" and "AUROC-0.5 (probes)" (Online Methods). Original DREAM challenge participants are indicated with their challenge 'Team' IDs. The highest score obtained for each evaluation criterion is in italics. Corr, Pearson correlation; AUROC (-0.5), area under the receiver operating characteristic curve (after subtracting 0.5). aAlgorithms were not subjected to data preprocessing improvement attempts either because they did not finish in the top four of the original DREAM challenge, are not fully automated or take greater than one CPU week to run on a single experiment. 
a

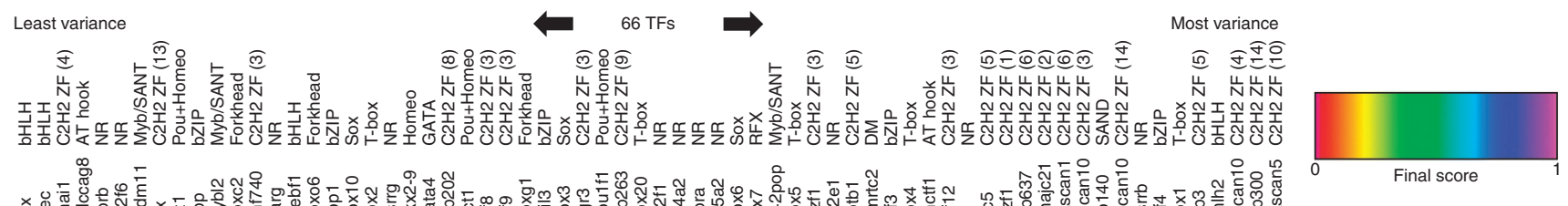

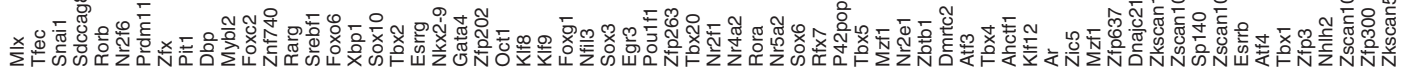

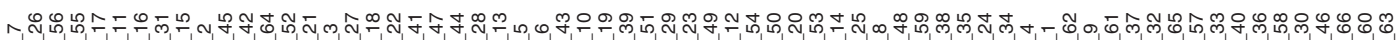

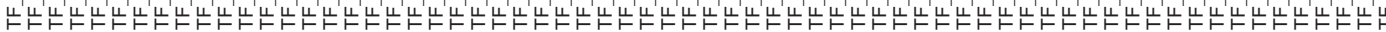
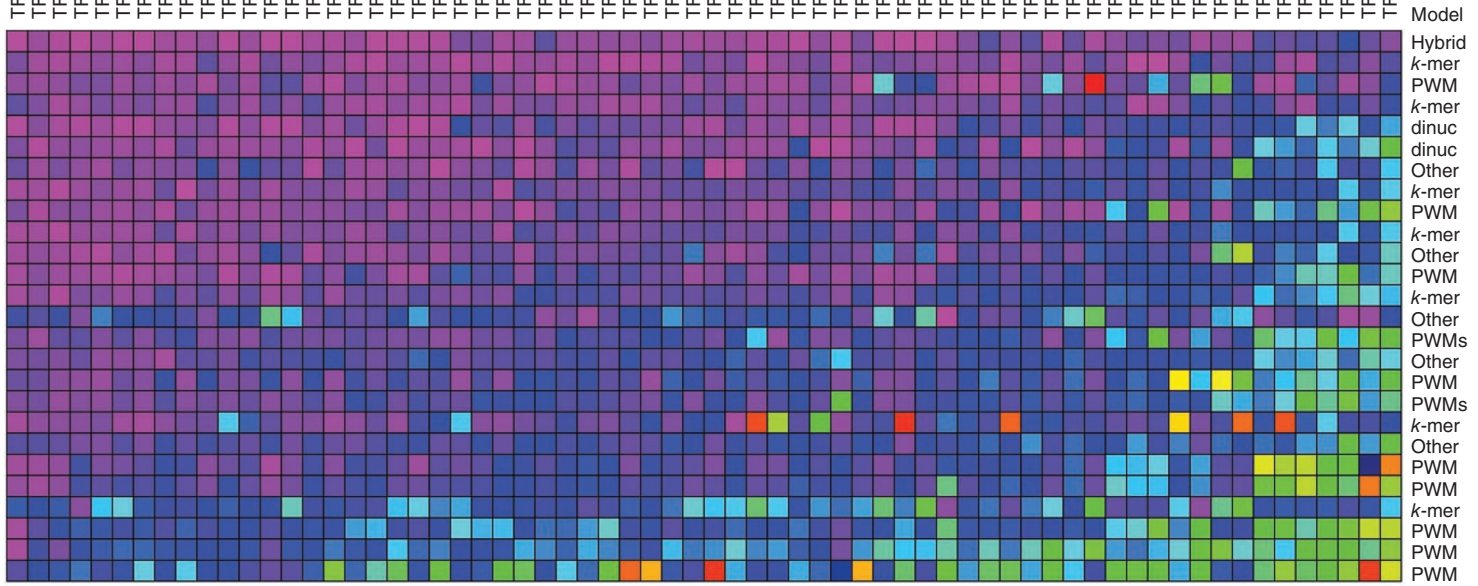

Algorithm

FeatureREDUCE

Team_D

Team_G

FeatureREDUCE dinuc

BEEML-PBM_dinuc

Team_F

8mer_pos

8 mer sum

8mer_sum

FeatureREDUCE_PWM

8 mer_max

Team_I

BEEML-PBM sec

Team_C

MatrixREDUCE

FeatureREDUCE_PWM_sec

Team_A

PWM_align

PWM_align_E

Team_K

Seed-And-Wobble

RankMotif++

Team_B

b

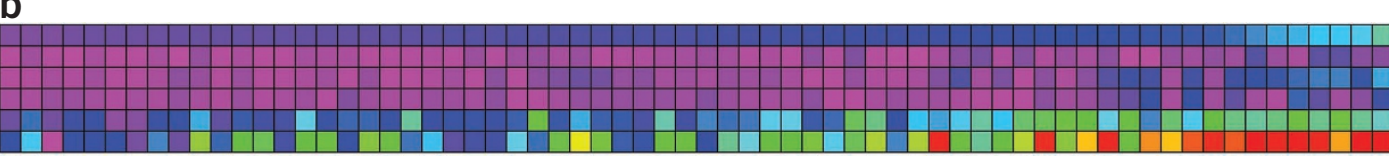

C

Statistic

Mea

Max (k-mer)

Max (dinuc)

Max (PWM)

Corr (Z)

Train $E>0.45$

0.3

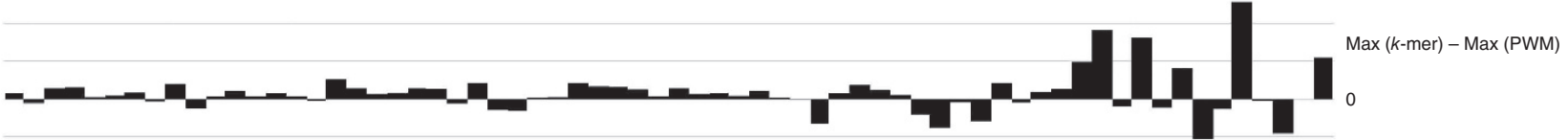

Figure 2 Comparison of algorithm performance by TF. (a) Final score of each algorithm for each TF. TF name, ID and family are depicted across the columns, and sequence specificity model type and name are depicted across the rows. Algorithms are sorted in decreasing order of final performance across all TFs. TFs are sorted in decreasing order of mean final score across all algorithms. Numbers in parentheses indicate the number of zinc fingers in the protein. (b) Summary statistics for each TF across all algorithms: mean final score, maximum final score achieved by any $k$-mer, dinucleotide or PWM-based algorithm, Pearson correlation of 8-mer Z-scores between replicate arrays, and the number of 8-mers with $E$-scores $>0.45$ on the training array (normalized by the maximum such value across all TFs). (c) Difference between the best score achieved by any $k$-mer-based algorithm and the best score achieved by any PWM-based algorithm for each TF.

algorithms, FeatureREDUCE and BEEML-PBM, both of which can be run using either type of model. Both did better overall when using the dinucleotide model (Table 2), although the difference was not dramatic, and certain TFs benefitted more than others (Supplementary Table 4; median improvement of 0.019 and 0.006 , respectively). In general, an overall improvement is not surprising because the dinucleotide model has more parameters. We note that the degree of improvement between FeatureREDUCE and BEEML-PBM is poorly correlated and is negatively correlated with how well each performs using only a mononucleotide PWM (Supplementary Fig. 3), suggesting that much of the improvement may be due to suboptimal mononucleotide PWMs. Of the six cases in which a dinucleotide model results in an improvement of $>5 \%$ in the final score for both FeatureREDUCE and BEEML-PBM, five are among the TFs for which it appears to be difficult to train a PWM (Fig. 2). These observations suggest that there are relatively few cases in which there are bona fide dinucleotide interactions that have a major impact on model performance.
Secondary motifs would represent alternative binding modes for a TF that are also not possible to capture with a single $\mathrm{PWM}^{7}$. The previously claimed widespread prevalence of secondary motifs ${ }^{7}$ was recently contested by the finding that a single BEEML-PBM PWM is more predictive than two PWMs derived by Seed-and-Wobble ${ }^{24}$, on the same data set used to support the original claim ${ }^{7}$. To more directly examine the importance of secondary motifs, we identified secondary motifs in both the PBM data of this study and that of the previous study ${ }^{7}$. We discovered secondary motifs by using the residuals of the primary motif probe signal intensity predictions for both BEEML-PBM and FeatureREDUCE, used regression on the training data to assign weights to the two motifs and evaluated their impact on the overall performance of each algorithm (Online Methods). The performance of both BEEML-PBM and FeatureREDUCE was, in fact, slightly weakened using this scheme (Table 2).

Because the decreased performance might be due to probe-level noise drowning out the comparatively weaker secondary motif signal, we evaluated the performance of the secondary motifs using 
Figure 3 Comparison of algorithm performance on in vivo data. For each algorithm, we trained a model (PWM, 2 PWMs, $k$-mer or dinucleotide) using PBM data, and gauged its ability to discriminate real from random ChIP peaks using the AUROC (Online Methods). Data for the first five TFs were taken from mouse ChIP-seq data. The final four are from yeast ChIP-exo data. The color scale is indicated at the bottom. Team_E was not run on the ChIP-exo data, because it requires initialization parameters specific to the individual TF. FeatureREDUCE was run using models of length 8 , instead of length 10 , owing to the superior performance of this length model on in vivo data (T.R.R. and H.J.B., unpublished data).

8-mer scores, using the newer 8-mer scoring procedure $^{24}$ (Online Methods). Under this scoring scheme, secondary motifs provided a slight increase in overall performance (2-8\% improvement in average correlation) (Supplementary Table 5). However, examination of secondary motif performance for each TF revealed that secondary motifs substantially increase performance only in specific cases (Supplementary Table 6). Moreover, as in the case of dinucleotidebased models, the degree of improvement in FeatureREDUCE and BEEML-PBM is poorly correlated, and again correlates negatively with how well each algorithm scores using only a mononucleotide PWM (Supplementary Fig. 3). Manual inspection of these examples revealed that improvement can typically be attributed to either the identification of a minor variation on the primary motif, a 'second chance' after producing an inaccurate motif on the first attempt, or by the identification of the second half-site for a TF that can bind DNA as a homodimer (Supplementary Note 6). We did identify several instances of what appear to be alternative binding modes, including three examples capturing the classic TAATA and ATGCWWW sequences of Pou+Homeodomain TFs, and extensions of primary motifs (e.g., extending the consensus sequence of $\mathrm{Nr} 5 \mathrm{a} 2$ from AAGGTCA to TCAAGGTCA), indicating that our methodology can detect bona fide cases of secondary motifs (Supplementary Note 6). Nonetheless, it appears as if the major benefit of secondary motifs is to make up for shortcomings in the initial motif-finding process.

\section{In vitro-derived PWMs accurately reflect in vivo binding}

We next asked whether conclusions reached using in vitro data also apply to TF binding in vivo. The sequence specificity of a TF is only one of several factors that determine where it binds in vivo (others include cofactors and DNA accessibility); nonetheless, motifs consistent with those obtained in vitro can often be derived directly from in vivo data ${ }^{7,30,31}$, indicating that the intrinsic sequence specificity of TFs is a major factor in controlling their DNA binding in vivo. We obtained publicly available ChIP-seq data for five of the mouse TFs whose DNA sequence preferences were measured using PBMs in this study, and ChIP-exo data from four yeast TFs whose preferences have been measured using PBMs in other studies. We then trained models on the PBM data using each algorithm and gauged their ability to accurately distinguish sequences bound by ChIP-seq and ChIP-exo peaks from control sequences. We also trained PWMs on the same in vivo data by running ChIPMunk ${ }^{32}$ and MEME-Chip ${ }^{33}$, methods that have been specifically tailored for motif discovery from ChIP-seq data, in a cross-validation setting. We evaluated each algorithm with AUROCs, which here measure the ability of a given algorithm to assign higher scores to positive (bound) sequences relative to control (random) sequences (Online Methods).

\begin{tabular}{|c|c|c|c|c|c|c|c|c|c|}
\hline Mean & Esrrb & Gata4 & Tbx20 & Tbx5 & Zfx & Gal4 & Phd1 & Rap1 & Reb1 \\
\hline 0.741 & 0.718 & 0.655 & 0.809 & 0.776 & 0.780 & 0.523 & 0.792 & 0.841 & 0.780 \\
\hline 0.725 & 0.684 & 0.726 & 0.631 & 0.679 & 0.753 & 0.785 & 0.723 & 0.770 & 0.780 \\
\hline 0.721 & 0.685 & 0.729 & 0.624 & 0.679 & 0.761 & 0.794 & 0.731 & 0.714 & 0.780 \\
\hline 0.703 & 0.688 & 0.726 & 0.663 & 0.699 & 0.798 & 0.761 & 0.732 & 0.849 & 0.416 \\
\hline 0.703 & 0.695 & 0.700 & 0.620 & 0.483 & 0.765 & 0.842 & 0.669 & 0.785 & 0.770 \\
\hline 0.695 & 0.698 & 0.702 & 0.618 & 0.473 & 0.763 & 0.769 & 0.680 & 0.788 & 0.770 \\
\hline 0.693 & 0.675 & 0.633 & 0.609 & 0.558 & 0.729 & 0.749 & 0.712 & 0.804 & 0.774 \\
\hline 0.681 & 0.625 & 0.725 & 0.529 & 0.683 & 0.805 & 0.781 & 0.727 & 0.703 & 0.558 \\
\hline 0.679 & 0.694 & 0.692 & 0.791 & 0.595 & 0.455 & 0.596 & 0.672 & 0.831 & 0.791 \\
\hline 0.678 & 0.703 & 0.736 & 0.661 & 0.675 & 0.793 & 0.761 & 0.552 & 0.726 & 0.495 \\
\hline 0.663 & 0.577 & 0.714 & 0.636 & 0.599 & 0.789 & $\mathrm{~N} / \mathrm{A}$ & N/A & $\mathrm{N} / \mathrm{A}$ & $\mathrm{N} / \mathrm{A}$ \\
\hline 0.653 & 0.699 & 0.637 & 0.627 & 0.582 & 0.704 & 0.733 & 0.720 & 0.611 & 0.564 \\
\hline 0.637 & 0.633 & 0.717 & 0.527 & 0.533 & 0.755 & 0.721 & 0.607 & 0.594 & 0.651 \\
\hline 0.630 & 0.511 & 0.666 & 0.609 & 0.423 & 0.669 & 0.749 & 0.733 & 0.680 & 0.633 \\
\hline 0.628 & 0.347 & 0.659 & 0.568 & 0.572 & 0.791 & 0.759 & 0.730 & 0.454 & 0.775 \\
\hline 0.610 & 0.677 & 0.744 & 0.573 & 0.716 & 0.803 & 0.382 & 0.731 & 0.411 & 0.453 \\
\hline 0.598 & 0.580 & 0.670 & 0.468 & 0.470 & 0.721 & 0.623 & 0.658 & 0.614 & 0.580 \\
\hline 0.567 & 0.496 & 0.603 & 0.415 & 0.425 & 0.717 & 0.631 & 0.675 & 0.572 & 0.575 \\
\hline
\end{tabular}

All PWM-based algorithms could discriminate ChIP-seq and ChIPexo peaks from control sequences to some degree, as evidenced by the fact that the average AUROC scores of all algorithms exceeded the random expectation of 0.5 (Fig. 3). Conversely, the algorithms that performed best in our in vitro evaluations (FeatureREDUCE and Team_D, which both incorporate $k$-mer sequence specificity models) perform poorly (Team_D) or substantially worse (FeatureREDUCE) in nearly all cases analyzed (as does the simple 8mer_sum algorithm; Fig. 3). Likewise, the dinucleotide versions of BEEML-PBM and FeatureREDUCE do not improve upon their PWM-based counterparts. The performance of the $k$-mer and dinucleotide-based in vitro-trained models on in vivo data could be due to a combination of modeling probe-specific effects such as GC content and complications arising from biases in genomic nucleotide content relative to PBM probe sequences. Indeed, the $8 \mathrm{mer}$ sum_high algorithm, which incorporates only 8-mers with $Z$-scores $>3$ (a cutoff that likely excludes PBM-specific background noise), performs substantially better than the 8 mer_sum algorithm, which incorporates scores across the entire range of $k$-mer values (Fig. 3).

Overall, PWMs produced by the FeatureREDUCE_PWM algorithm perform best on in vivo data (Fig. 3). Notably, FeatureREDUCE_PWM performs similarly to ChIPMunk, and out-performs the MEME-Chip algorithm, despite the fact that the latter algorithms trained their PWMs on the ChIP-seq data, and should thus incorporate features unique to in vivo data, such as nucleotide bias. All of our conclusions were robust to a variety of positive and negative sequence settings (Supplementary Table 7). Thus, at least for the nine TFs we examined here, in vitro-derived PWMs are in general better than in vitro-derived $k$-mer and dinucleotide models, and similar to in vivo-derived PWMs, in terms of predicting bound versus unbound ChIP-seq and ChIP-exo sequences.

\section{Accurate prediction of data from alternative in vitro assays}

Finally, we examined how well PBM-derived motifs, with or without dinucleotides, secondary motifs or $k$-mers, could predict data for 24 TFs that have been assayed using the MITOMI 916 or HiTS-FLIP technologies ${ }^{8}$, all of which also have PBM data available from other studies $^{7,31,34}$. We trained the best-performing FeatureREDUCE algorithm on the PBM data in each of its possible settings: PWM only, 2 PWMs (secondary motifs), dinucleotides and dinucleotides $+k$-mers. We then compared the ability of each model to predict the values produced by the other technology.

The inclusion of features beyond mononucleotide PWMs had limited impact on the majority of these 24 TFs (Supplementary Note 7). 


\section{Box 1 Appearance and information content of a motif may not reflect its accuracy}

Sequence $\log _{0} 39,40$ provide a simple, intuitive means for conveying information about a TF's binding preferences. However, several aspects of their interpretation can be misleading. To illustrate, logos produced by the eight PWMbased algorithms evaluated here are depicted for TF_6, the $\mathrm{C}_{2} \mathrm{H}_{2}$ zinc finger TF KIf9 (Fig. 4). At a glance, the PWMs produced by Seed-andWobble and the PWM_align algorithms might be interpreted as being superior to the others, given their high information content. However, based on our evaluations, these PWMs are in fact too stringent, and place too much emphasis on the consensus sequence of this TF (compare the final scores of each algorithm). Rather, the lower information motif produced by BEEML-PBM is a better predictor of KIf9's sequence preferences. In general, this observation holds for almost all TFs analyzed here-the Seed-and-Wobble and the PWM_align algorithms tend to produce PWMs that are 'too stringent' and too long, and energy-based algorithms such as BEEML-PBM produce motifs that represent the correct degree of degeneracy and length (see Supplementary Fig. 4 for logos and Fig. 2 for evaluations).

Similarly, different interpretations might be made about a TF's sequence preferences based on which visualization method is used to depict a PWM. For example, the importance of the initial $T$ nucleotide in the TAACGG consensus sequence in the motifs of BEEML-PBM might be considered negligible upon viewing of the information

content-based logo, whereas this nucleotide would likely be considered highly important based on the frequency logo. Indeed, the information specified at this position does play a large role in the overall effectiveness of the motif. When ignoring the frequencies specified at this position (that is, setting all four nucleotide frequencies to 0.25 ), the correlation between BEEML-PBM's predicted and actual probe signal intensities drops from 0.58 to 0.38 . Furthermore, the sequence logos for BEEML-PBM, MatrixREDUCE, FeatureREDUCE and Team_E appear nearly indistinguishable based on the sequence logos, despite their drastically differing final evaluation scores. In summary, we find that the appearance of sequence logos has little bearing on their predictive accuracy.
We note, however, that we detected specific examples where more complicated models provided an increase in performance across platforms (Supplementary Note 7 ). For example, $k$-mers and secondary motifs both improve cross-platform performance for Cbf1. This finding confirms that PBMs are capable of detecting cases where more complicated binding modes exist, and that these models are capable of improving predictive performance on other data sources. Taken together, these results are consistent with our findings that PWMs work well for most TFs, although certain TFs require more complicated models.

\section{DISCUSSION}

We have come to several major conclusions on the basis of this study, which have broad implications for the representation of sequence specificity of DNA-binding proteins. We note that the exact conclusions reached depend on both the TFs used for evaluation and the evaluation criteria, a fact that likely accounts for the ongoing controversy in this area. However, our general conclusions are robust to changes in the evaluation procedure. In addition, our conclusion that wellimplemented PWMs can perform as effectively as more complicated models in most cases is supported by cross-technology analysis of in vitro data and by analysis of in vivo data.
Our first major conclusion is that, when testing on PBM data, $k$-mer-based models score best overall. Other approaches do nearly as well, however, and details of implementation, such as parameter estimation techniques, can be as important to the performance of an algorithm as the underlying model. Indeed, the algorithms that produce the most predictive PWMs, FeatureREDUCE_PWM and BEEML-PBM, which both train PWMs in an energy-based framework (Supplementary Note 8), perform similarly to more complicated models for the majority of TFs, supporting the contention that imperfections in motif derivation (and scoring) underlie most of the apparent superiority of $k$-mer scoring that we previously reported ${ }^{7,24}$. PWMs consistently fared poorly in $\sim 10 \%$ of the TFs, relative to $k$-mer-based sequence specificity models; however, many of these cases are characterized by having few high-scoring 8-mers (Fig. 2b and Supplementary Note 4). Thus, the scarcity of the data itself may limit the ability of algorithms to train a PWM. Modification of the algorithms may help improve these cases.

The fact that incorporation of dinucleotide interactions improves the performance of both BEEML-PBM and FeatureREDUCE, but for different sets of TFs, suggests that the need for these extensions to mononucleotide PWM is driven more by the algorithm than by a 
property of the TF. Dinucleotide interactions clearly do exist ${ }^{25}$ and were highlighted in previous analyses using MITOMI $^{9}$, HiTS-FLIP $^{8}$ and $\mathrm{PBM}^{19}$ data. However, these studies did not specifically ask how much of the overall variation in the data (e.g., using Pearson correlation) is accounted for by mononucleotide versus dinucleotide PWMs. We also note that more complex models can be more prone to learning platform-specific noise. At present it is not clear what the best approach is for different platforms; resolving the source and relative contribution of complexities in DNA-binding data would benefit from analysis of the same TFs on multiple high-resolution platforms.

One striking outcome of our study is that the appearance and information content of a motif has little bearing on its accuracy. The motifs produced by BEEML-PBM and FeatureREDUCE_PWM-two of the highest-scoring PWM algorithms-are, in general, those with the lowest information content (Box 1, Fig. 4 and Supplementary Fig. 4). Conversely, PWMs produced by Seed-and-Wobble and PWM_align appear to be the strongest (that is, they are wider and have larger letters in the traditional 'information content' sequence logos), but they score substantially lower than those of BEEML-PBM and FeatureREDUCE_PWM, on both PBM and ChIP-seq data. We conclude from this analysis that information content has little to do with the accuracy and utility of a motif, underscoring the fact that degeneracy is common among eukaryotic TF sequence specificities, and that most TFs will bind to many variations of their 'consensus sequence', albeit at lower affinity. Indeed, previous studies have demonstrated the importance of low affinity binding sites in vivo ${ }^{35-38}$. PWMs that allow for a greater amount of degeneracy (and hence have lower information content) are able to better capture the full range of lower affinity sites.

The finding that different algorithms excel (and fail) for different TFs suggests that an algorithm incorporating all of their advantages will likely outperform any individual one. To aid in the continued improvement of algorithms for the modeling of TF binding specificities, we have created a web server that allows users to upload their own probe intensity predictions, and compare them to those of the algorithms evaluated here (http://www.ebi.ac.uk/saezrodriguez-srv/ d5c2/cgi-bin/TF_web.pl). We anticipate that the availability of this resource will help encourage future improvements to algorithms for the modeling and prediction of TF binding specificities.

\section{METHODS}

Methods and any associated references are available in the online version of the paper.

Data availability. PBM data, GEO: GSE42864. The data are also available on the project website (http://hugheslab.ccbr.utoronto.ca/ supplementary-data/DREAM5/). PWMs and algorithm source code are found in Supplementary Data 1 and 2.

Note: Supplementary information is available in the online version of the paper.

\section{ACKNOWLEDGMENTS}

We thank H. van Bakel and M. Albu for database assistance, and members of the Hughes laboratory for helpful discussion. M.T.W. was supported by fellowships from the Canadian Institutes of Health Research (CIHR) and the Canadian Institute for Advanced Research (CIFAR) Junior Fellows Genetic Networks Program. This work was supported in part by the Ontario Research Fund and Genome Canada through the Ontario Genomics Institute, and the March of Dimes (T.R.H.). Funding was also provided by Operating Grant MOP-77721 from CIHR to T.R.H. and M.L.B., and grant no. R01 HG003985 from the US National Institutes of Health/National Human Genome Research Institute to M.L.B., as well as US National Institutes of Health grants R01HG003008 and U54CA121852 and a John Simon Guggenheim Foundation Fellowship to H.J.B. M.A., K.L., H.L. and M.L. were supported by the Academy of Finland (project 260403) and EU ERASysBio ERA-NET. Y.O., C.L. and R.S. were funded by the European Community's Seventh Framework Programme under grant agreement no. HEALTH-F4-2009-223575 for the TRIREME project, and by the Israel Science Foundation (grant no. 802/08). Y.O. was supported in part by a fellowship from the Edmond J. Safra Bioinformatics Program at Tel Aviv University. J.G., I.G., S.P. and J.K. were supported by grant XP3624HP/0606T by the Ministry of Culture of Saxony-Anhalt. A.M. was supported by US National Science Foundation (NSF) grant PHY-1022140. C.C. was supported by NSF grant PHY-0957573. J.B.K. was supported by the Simons Center for Quantitative Biology at Cold Spring Harbor Laboratory.

\section{AUTHOR CONTRIBUTIONS}

M.T.W. and T.R.H. wrote the manuscript. T.R.H., M.T.W., M.L.B. and A.V. conceived of the study. M.T.W. did the majority of the computational analyses. M.A., Y.Z. and T.R.R. did additional computational analyses. A.C. and S.T, performed the PBM experiments. T.R.H., M.T.W., G.S. and R.N. designed and carried out the DREAM5 TF challenge. The DREAM5 Consortium and M.A. participated in the DREAM5 TF challenge. R.N., J.S.-R., T.C. and M.T.W. designed and created the prediction server. M.L.B., G.S., Q.D.M. and H.J.B. provided critical feedback on the manuscript.

\section{COMPETING FINANCIAL INTERESTS}

The authors declare no competing financial interests.

Published online at http://www.nature.com/doifinder/10.1038/nbt.2486. Reprints and permissions information is available online at http://www.nature.com/ reprints/index.html.

1. Stormo, G.D., Schneider, T.D., Gold, L. \& Ehrenfeucht, A. Use of the 'Perceptron' algorithm to distinguish translational initiation sites in E. coli. Nucleic Acids Res. 10, 2997-3011 (1982)

2. Berg, O.G. \& von Hippel, P.H. Selection of DNA binding sites by regulatory proteins. Statistical-mechanical theory and application to operators and promoters. J. Mol. Biol. 193, 723-743 (1987)

3. Stormo, G.D. Consensus patterns in DNA. Methods Enzymol. 183, 211-221 (1990).

4. Siddharthan, R. Dinucleotide weight matrices for predicting transcription factor binding sites: generalizing the position weight matrix. PLOS ONE 5, e9722 (2010).

5. Zhao, X., Huang, H. \& Speed, T.P. Finding short DNA motifs using permuted Markov models. J. Comput. Biol. 12, 894-906 (2005).

6. Sharon, E., Lubliner, S. \& Segal, E. A feature-based approach to modeling proteinDNA interactions. PLOS Comput. Biol. 4, e1000154 (2008).

7. Badis, G. et al. Diversity and complexity in DNA recognition by transcription factors. Science 324, 1720-1723 (2009)

8. Nutiu, R. et al. Direct measurement of DNA affinity landscapes on a high-throughput sequencing instrument. Nat. Biotechnol. 29, 659-664 (2011).

9. Maerkl, S.J. \& Quake, S.R. A systems approach to measuring the binding energy landscapes of transcription factors. Science 315, 233-237 (2007).

10. Agius, P., Arvey, A., Chang, W., Noble, W.S. \& Leslie, C. High resolution models of transcription factor-DNA affinities improve in vitro and in vivo binding predictions. PLoS Comput. Biol. 6, e1000916 (2010).

11. Annala, M., Laurila, K., Lähdesmäki, H. \& Nykter, M. A linear model for transcription factor binding affinity prediction in protein binding microarrays. PLOS ONE 6 , e20059 (2011).

12. Zhao, Y., Granas, D. \& Stormo, G.D. Inferring binding energies from selected binding sites. PLOS Comput. Biol. 5, e1000590 (2009).

13. Slattery, M. et al. Cofactor binding evokes latent differences in DNA binding specificity between Hox proteins. Cell 147, 1270-1282 (2011).

14. Jolma, A. et al. Multiplexed massively parallel SELEX for characterization of human transcription factor binding specificities. Genome Res. 20, 861-873 (2010).

15. Zykovich, A., Korf, I. \& Segal, D.J. Bind-n-Seq: high-throughput analysis of in vitro protein-DNA interactions using massively parallel sequencing. Nucleic Acids Res. 37, e151 (2009).

16. Fordyce, P.M. et al. De novo identification and biophysical characterization of transcription-factor binding sites with microfluidic affinity analysis. Nat. Biotechnol. 28, 970-975 (2010).

17. Warren, C.L. et al. Defining the sequence-recognition profile of DNA-binding molecules. Proc. Natl. Acad. Sci. USA 103, 867-872 (2006).

18. Meng, X., Brodsky, M.H. \& Wolfe, S.A. A bacterial one-hybrid system for determining the DNA-binding specificity of transcription factors. Nat. Biotechnol. 23, 988-994 (2005).

19. Berger, M.F. et al. Compact, universal DNA microarrays to comprehensively determine transcription-factor binding site specificities. Nat. Biotechnol. 24, 1429-1435 (2006).

20. Stormo, G.D. \& Zhao, Y. Determining the specificity of protein-DNA interactions. Nat. Rev. Genet. 11, 751-760 (2010).

21. Prill, R.J. et al. Towards a rigorous assessment of systems biology models: the DREAM3 challenges. PLOS ONE 5, e9202 (2010). 
22. Stolovitzky, G., Monroe, D. \& Califano, A. Dialogue on reverse-engineering assessment and methods: the DREAM of high-throughput pathway inference. Ann. NY Acad. Sci. 1115, 1-22 (2007).

23. Stolovitzky, G., Prill, R.J. \& Califano, A. Lessons from the DREAM2 Challenges. Ann. NY Acad. Sci. 1158, 159-195 (2009).

24. Zhao, Y. \& Stormo, G.D. Quantitative analysis demonstrates most transcription factors require only simple models of specificity. Nat. Biotechnol. 29, 480-483 (2011)

25. Zhao, Y., Ruan, S., Pandey, M. \& Stormo, G.D. Improved models for transcription factor binding site identification using non-independent interactions. Genetics 191, 781-790 (2012)

26. Foat, B.C., Morozov, A.V. \& Bussemaker, H.J. Statistical mechanical modeling of genome-wide transcription factor occupancy data by MatrixREDUCE. Bioinformatics 22, e141-e149 (2006)

27. Chen, X., Hughes, T.R. \& Morris, Q. RankMotif.: a motif-search algorithm that accounts for relative ranks of K-mers in binding transcription factors. Bioinformatics 23, i72-i79 (2007).

28. Berger, M.F. et al. Variation in homeodomain DNA binding revealed by high-resolution analysis of sequence preferences. Cell 133, 1266-1276 (2008)

29. Rhee, H.S. \& Pugh, B.F. Comprehensive genome-wide protein-DNA interactions detected at single-nucleotide resolution. Cell 147, 1408-1419 (2011).

30. Wei, G.H. et al. Genome-wide analysis of ETS-family DNA-binding in vitro and in vivo. EMBO J. 29, 2147-2160 (2010).

31. de Boer, C.G. \& Hughes, T.R. YeTFaSCo: a database of evaluated yeast transcription factor sequence specificities. Nucleic Acids Res. 40, D169-D179 (2012).

32. Kulakovskiy, I.V., Boeva, V.A., Favorov, A.V. \& Makeev, V.J. Deep and wide digging for binding motifs in ChIP-Seq data. Bioinformatics 26, 2622-2623 (2010).

33. Machanick, P. \& Bailey, T.L. MEME-ChIP: motif analysis of large DNA datasets. Bioinformatics 27, 1696-1697 (2011).

34. Zhu, C. et al. High-resolution DNA-binding specificity analysis of yeast transcription factors. Genome Res. 19, 556-566 (2009).
35. John, S., Marais, R., Child, R., Light, Y. \& Leonard, W.J. Importance of low affinity Elf- 1 sites in the regulation of lymphoid-specific inducible gene expression. J. Exp. Med. 183, 743-750 (1996).

36. Tanay, A. Extensive low-affinity transcriptional interactions in the yeast genome. Genome Res. 16, 962-972 (2006).

37. Jaeger, S.A. et al. Conservation and regulatory associations of a wide affinity range of mouse transcription factor binding sites. Genomics 95, 185-195 (2010).

38. Segal, E., Raveh-Sadka, T., Schroeder, M., Unnerstall, U. \& Gaul, U. Predicting expression patterns from regulatory sequence in Drosophila segmentation. Nature 451, 535-540 (2008)

39. Schneider, T.D. \& Stephens, R.M. Sequence logos: a new way to display consensus sequences. Nucleic Acids Res. 18, 6097-6100 (1990).

40. Crooks, G.E., Hon, G., Chandonia, J.M. \& Brenner, S.E. WebLogo: a sequence logo generator. Genome Res. 14, 1188-1190 (2004).

41. Keilwagen, J. et al. De-novo discovery of differentially abundant transcription factor binding sites including their positional preference. PLOS Comput. Biol. 7, e1001070 (2011).

42. Bailey, T.L. \& Elkan, C. Fitting a mixture model by expectation maximization to discove motifs in biopolymers. Proc. Int. Conf. Intell. Syst. Mol. Biol. 2, 28-36 (1994).

43. Schutz, F. \& Delorenzi, M. MAMOT: hidden Markov modeling tool. Bioinformatics 24, 1399-1400 (2008)

44. Kinney, J.B., Tkacik, G. \& Callan, C.G. Jr. Precise physical models of protein-DNA interaction from high-throughput data. Proc. Natl. Acad. Sci. USA 104, 501-506 (2007).

45. Kinney, J.B., Murugan, A., Callan, C.G. Jr. \& Cox, E.C. Using deep sequencing to characterize the biophysical mechanism of a transcriptional regulatory sequence. Proc. Natl. Acad. Sci. USA 107, 9158-9163 (2010).

46. Linhart, C., Halperin, Y. \& Shamir, R. Transcription factor and microRNA moti discovery: the Amadeus platform and a compendium of metazoan target sets. Genome Res. 18, 1180-1189 (2008).

47. Tibshirani, R. Regression shrinkage and selection via the Lasso. J. R. Stat. Soc. B 58, 267-288 (1996)

48. Chen, C.Y. et al. Discovering gapped binding sites of yeast transcription factors. Proc. Natl. Acad. Sci. USA 105, 2527-2532 (2008).

\section{The complete list of DREAM5 Consortium authors is as follows:}

\section{Phaedra Agius ${ }^{13}$, Aaron Arvey ${ }^{13}$, Philipp Bucher ${ }^{14,15}$, Curtis G Callan Jr ${ }^{16,17}$, Cheng Wei Chang ${ }^{18}$, Chien-Yu Chen ${ }^{19}$, Yong-Syuan Chen ${ }^{19}$, Yu-Wei Chu ${ }^{19,20}$, Jan Grau ${ }^{21}$, Ivo Grosse ${ }^{21}$, Vidhya Jagannathan ${ }^{14,22}$, Jens Keilwagen ${ }^{23}$, Szymon M Kiełbasa ${ }^{24}$, Justin B Kinney ${ }^{25}$, Holger Klein ${ }^{26}$, Miron B Kursa ${ }^{27}$, Harri Lähdesmäki ${ }^{28,29}$, Kirsti Laurila ${ }^{30}$, Chengwei Lei ${ }^{31}$, Christina Leslie ${ }^{13}$, Chaim Linhart ${ }^{32}$, Anand Murugan ${ }^{16}$, Alena Myšičková ${ }^{24}$, William Stafford Noble ${ }^{33}$, Matti Nykter ${ }^{30}$, Yaron Orenstein ${ }^{32}$, Stefan Posch ${ }^{21}$, Jianhua Ruan ${ }^{31}$, Witold R Rudnicki ${ }^{27}$, Christoph D Schmid ${ }^{14,34,35}$, Ron Shamir ${ }^{32}$, Wing-Kin Sung ${ }^{18,36}$, Martin Vingron ${ }^{24}$ \& Zhizhuo Zhang ${ }^{36}$}

\footnotetext{
${ }^{13}$ Computational Biology Program, Sloan-Kettering Institute, Memorial Sloan-Kettering Cancer Center, New York, New York, USA. ${ }^{14}$ Swiss Institute of Bioinformatics, Lausanne, Switzerland. 15EPFL (École Polytechnique Fédérale de Lausanne) SV ISREC (The Swiss Institute for Experimental Cancer Research) GR-BUCHER, Lausanne, Switzerland. ${ }^{16}$ Department of Physics, Princeton University, Princeton, New Jersey, USA. ${ }^{17}$ Lewis-Sigler Institute for Integrative Genomics, Princeton University, Princeton, New Jersey, USA. ${ }^{18}$ Genome Institute of Singapore, Singapore. ${ }^{19}$ Department of Bio-Industrial Mechatronics Engineering, National Taiwan University, Taipei, Taiwan. ${ }^{20}$ Graduate Institute of Communication Engineering, National Taiwan University, Taipei, Taiwan. ${ }^{21}$ Institute of Computer Science, Martin Luther University, Halle-Wittenberg, Germany. ${ }^{22}$ Institute for Genetics, University of Bern, Bern, Switzerland. ${ }^{23}$ Leibniz Institute of Plant Genetics and Crop Plant Research, Gatersleben, Germany. ${ }^{24}$ Max Planck Institute for Molecular Genetics, Berlin, Germany. ${ }^{25}$ Simons Center for Quantitative Biology, Cold Spring Harbor Laboratory, Cold Spring Harbor, New York, USA. ${ }^{26}$ MicroDiscovery GmbH, Berlin, Germany. ${ }^{27}$ Interdisciplinary Centre for Mathematical and Computational Modelling, University of Warsaw, Warsaw, Poland. ${ }^{28}$ Department of Information and Computer Science, Aalto University School of Science and Technology, Aalto, Finland. ${ }^{29}$ Turku Centre for Biotechnology, Turku University, Turku, Finland. ${ }^{30}$ Department of Signal Processing, Tampere University of Technology, Tampere, Finland. ${ }^{31}$ Department of Computer Science, University of Texas at San Antonio, San Antonio, Texas, USA. ${ }^{32}$ Blavatnik School of Computer Science, Tel Aviv University, Tel Aviv, Israel. ${ }^{33}$ Department of Genome Sciences, University of Washington, Seattle, Washington, USA. ${ }^{34}$ Swiss Tropical and Public Health Institute (Swiss TPH), Basel, Switzerland. ${ }^{35}$ University of Basel, Basel, Switzerland. ${ }^{36}$ School of Computing, National University of Singapore, Singapore.
} 


\section{ONLINE METHODS}

Protein binding microarray experiments. Details of the design and use of PBMs have been described elsewhere ${ }^{19,28,49,50}$. Here, we used two different universal $\mathrm{PBM}$ array designs, designated 'ME' and ' $\mathrm{HK}$ ', after the initials of their designers. Information about individual plasmids is available in Supplementary Table 8 . We identified the DNA binding domain (DBD) of each TF by searching for Pfam domains ${ }^{51}$ using the HMMER tool ${ }^{52}$. DBD sequences along with 50 amino acid residues on either side of the DBD in the native protein were cloned as SacI-BamHI fragments into pTH5325, a modified T7-driven glutathione S-transferase (GST) expression vector. Briefly, we used $150 \mathrm{ng}$ of plasmid DNA in a $15 \mu \mathrm{l}$ in vitro transcription and/or translation reaction using a PURExpress In Vitro Protein Synthesis Kit (New England BioLabs) supplemented with RNase inhibitor and $50 \mu \mathrm{M}$ zinc acetate. After a 2-h incubation at $37{ }^{\circ} \mathrm{C}, 12.5 \mathrm{ml}$ of the mix was added to $137.5 \mathrm{ml}$ of protein-binding solution for a final mix of $\mathrm{PBS} / 2 \%$ skim milk/ $0.2 \mathrm{mg}$ per $\mathrm{ml}$ BSA $/ 50 \mu \mathrm{M}$ zinc acetate $/ 0.1 \%$ Tween- 20 . This mixture was added to an array previously blocked with $\mathrm{PBS} / 2 \%$ skim milk and washed once with $\mathrm{PBS} / 0.1 \%$ Tween-20 and once with PBS/0.01\% Triton-X 100. After a 1-h incubation at room temperature, the array was washed once with PBS/0.5\% Tween-20/ $50 \mathrm{mM}$ zinc acetate and once with PBS/0.01\% Triton-X 100/50 mM zinc acetate. Cy5-labeled anti-GST antibody was added, diluted in PBS/2\% skim milk/50 mM zinc acetate. After a 1-h incubation at room temperature, the array was washed three times with PBS/0.05\% Tween-20/50 mM zinc acetate and once with PBS/50 mM zinc acetate. The array was then imaged using an Agilent microarray scanner at $2 \mu \mathrm{m}$ resolution. Images were scanned at two power settings: $100 \%$ photomultiplier tube (PMT) voltage (high), and 10\% PMT (low). The two resulting grid images were then manually examined, and the scan with the fewest number of saturated spots was used. Image spot intensities were quantified using ImaGene software (BioDiscovery).

Prediction of array intensities. We evaluated a panel of 26 algorithms, based on their ability to accurately predict array intensities (Table 1). Parameters used for the published and novel algorithms and full descriptions of the algorithms submitted as part of the DREAM5 challenge can be found in Supplementary Note 9.

Evaluation criteria. We evaluated the probe intensity predictions produced by each algorithm for each TF using two evaluation criteria (see Fig. 1 for illustrations, and below for descriptions). Before performing our evaluations, we removed all spots manually flagged as bad or suspect from the set of test probe intensities used in the evaluations. Each of the 66 experiments was scored individually using each criterion. The final score for both criteria was calculated as the average across all 66 experiments. To assign a final score to each algorithm, the score distributions of both of the criteria were first converted to relative scores, such that the best-performing algorithm for the given criterion received a score of 1 , and the scores of all other algorithms were relative to this best score (e.g., 0.90 as good as the top score, 0.80 as good). The final score for each algorithm was then calculated as the average of its two relative score, and can hence be interpreted as how well the algorithm performed relative to the best algorithm, on average. A similar calculation was done to achieve the final scores of the individual TFs depicted in Figure 2. In this case, the calculations were carried out as described above, but individually for each of the 66 experiments (that is, skipping the step of averaging across all 66 experiments).

Pearson correlation of probe intensities. We measured the correlation between the predicted probe intensities $p$ and the actual intensities $a$ using the (centered) Pearson correlation, $r$ :

$$
r(p, a)=\frac{\sum_{i=1}^{N}\left(p_{i}-\bar{p}\right)\left(a_{i}-\bar{a}\right)}{\sqrt{\sum_{i=1}^{N}\left(p_{i}-\bar{p}\right)^{2} \sum_{i=1}^{N}\left(a_{i}-\bar{a}\right)^{2}}}
$$

where $N$ is the total number of probe sequences on the array, $\bar{p}$ indicates the mean probe intensity across all predicted probe intensities, and $\bar{a}$ indicates the mean across all actual probe intensities. We chose not to use the Spearman correlation because its rank transformation results in a loss of resolution in the high probe intensity range, placing greater emphasis on the (majority of) unbound, low intensity probes.

AUROC of probe intensity predictions. As a second measure of an algorithm's accuracy, we quantified the ability of the given algorithm to assign high ranks to bright probes. We defined bright probes as those whose intensities were 4 standard deviations above the mean in the actual experiment ${ }^{27}$. This results in an average of 350 bright probes per experiment, with an enforced minimum of 50 , and a maximum of 1,300. For each algorithm's predictions for each TF, we ranked the $\sim 40,000$ probes based on their predicted intensities and calculated the AUROC of the actual bright probes. We subtracted 0.50 from the final AUROC score, so that a value of 0 corresponds to random expectation.

Identification and evaluation of secondary motifs. We identified primary and secondary PWMs for each TF in this paper and a set of previously published $\mathrm{TFs}^{7}$ using two of the top algorithms (FeatureREDUCE and BEEML-PBM), and used a combination of both PWMs to predict probe intensities using the following procedure:

1. Run the algorithm to train a single $\mathrm{PFM}, \mathrm{PFM}_{1}$, on the training array data.

2. Use $P M_{1}$ to predict the probe intensities of the training array (intensities $)_{1}$ ).

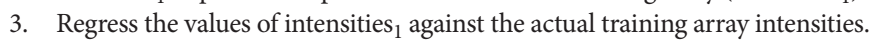

4. Calculate the residuals by subtracting the regressed intensities from the actual training array intensities. Set any resulting negative values to 0 .

5. Run the algorithm to train a single $\mathrm{PFM}, \mathrm{PFM}_{2}$, on the residuals.

6. Use $\mathrm{PFM}_{2}$ to predict the probe sequences of the training array (intensities $)_{2}$ ).

7. Regress the two sets of probe scores (intensities ${ }_{1}$ and intensities ${ }_{2}$ ) against the training probe intensities to learn the weights of the two PFMs.

8. Use $\mathrm{PFM}_{1}$ to predict the probe intensities of the test array.

9. Use $\mathrm{PFM}_{2}$ to predict the probe intensities of the test array.

10. Combine the two sets of predicted probe intensities using the regression coefficients learned on the training array in step 7.

We found that the resulting secondary motif probe intensity predictions decreased performance for both algorithms in our evaluation scheme (Table 2). We therefore tried an alternative scheme ${ }^{24}$ where we converted the training intensities and probe intensity predictions of $\mathrm{PFM}_{1}$ and $\mathrm{PFM}_{2}$ to 8-mers (using the median probe intensity), and then learned the weights of the two PWMs by performing regression on these 8 -mer values. The resulting weights were then used to combine the predicted 8-mer scores of $\mathrm{PWM}_{1}$ and $\mathrm{PWM}_{2}$ on the test data. Using this strategy, we observed a minor increase in overall performance for both algorithms on both data sets (Supplementary Table 6).

Comparison of algorithm performance on in vivo data. We gauged the ability of each algorithm to predict in vivo TF binding by comparing the ability of their models to accurately predict ChIP-seq and ChIP-exo binding data. We searched for publicly available ChIP-seq data measuring the in vivo binding of any of the 66 mouse TFs evaluated here using a variety of sources, including the hmCHIP database ${ }^{53}$, ArrayExpress ${ }^{54}$ and the NCBI Gene Expression Omnibus ${ }^{55}$. Some data was unusable because scores were not assigned to individual peak calls. In total, we obtained data for five TFs: Esrrb (GEO accession GSM288355), Zfx (GEO accession GSM288352), Tbx20 (GEO accession GSM734426), Tbx5 (GEO accession GSM558908) and Gata4 (GEO accession GSM558904). We also obtained four yeast ChIP-exo experiments from the literature ${ }^{29}$.

For each in vivo data set, we defined a set of positive (bound) sequences and negative (control) sequences. Positive sequences were defined for ChIPseq data as the 500 highest-confidence peaks, using only the middle 100 bases of each peak (similar results were obtained when using the middle 50 bases; Supplementary Table 7). Full-length sequence reads were used for ChIP-exo data. Random sequences were defined in one of three ways: (i) 500 randomly chosen genomic regions of the same length as the positive sequences, excluding all repeat sequences using RepeatMasker; (ii) 500 sequences of length 100 (or 50) randomly chosen from promoter sequences, where promoters were 
defined as the 5,000 base upstream regions upstream of the transcription start site of Ref-seq genes, excluding all sequences flagged by RepeatMasker (obtained from the UCSC Genome Browser ${ }^{56}$ ); (iii) 500 randomly shuffled positive sequences, where dinucleotide frequencies were maintained.

We assessed the PWMs produced by each algorithm by scoring the positive and negative sequences, and calculating the AUROC of the sequence scores using the positive and negative probe labels. Positive and negative ChIP sequences were scored using the energy scoring framework of BEEML-PBM (setting mu to 0 , and ignoring strand-specific biases). The final score for each algorithm on each TF was calculated as the mean AUROC across the three negative peaks sets. We also scored the probe sequences using the $k$-mer-based algorithms of Team_D, 8mer_sum, and FeatureREDUCE, and the dinucleotide algorithms of BEEML-PBM_dinuc and FeatureREDUCE_dinuc. We examined the performance of BEEML-PBM and FeatureREDUCE secondary motifs on the in vivo data using the PWMs and PWM weights learned from the in vitro data, as described above. To compare the in vitro generated motifs to in vivo-derived ones, we also used PWMs derived by ChIPMunk ${ }^{32}$ and MEME-Chip ${ }^{33}$ when run on the same in vivo data in a cross-validation setting. For these analyses, half of the positive probes were randomly chosen for training, and the other half were used for testing. This procedure was applied ten times, and the final numbers reported are the average evaluation scores across all ten iterations.

49. Philippakis, A.A., Qureshi, A.M., Berger, M.F. \& Bulyk, M.L. Design of compact, universal DNA microarrays for protein binding microarray experiments. J. Comput. Biol. 15, 655-665 (2008)

50. Lam, K.N., van Bakel, H., Cote, A.G., van der Ven, A. \& Hughes, T.R. Sequence specificity is obtained from the majority of modular $\mathrm{C} 2 \mathrm{H} 2$ zinc-finger arrays. Nucleic Acids Res. 39, 4680-4690 (2011).

51. Finn, R.D. et al. The Pfam protein families database. Nucleic Acids Res. 38 D211-D222 (2010)

52. Eddy, S.R. A new generation of homology search tools based on probabilistic inference. Genome Inform. 23, 205-211 (2009).

53. Chen, L., Wu, G. \& Ji, H. hmChIP: a database and web server for exploring publicly available human and mouse ChIP-seq and ChIP-chip data. Bioinformatics 27, 1447-1448 (2011).

54. Parkinson, H. et al. ArrayExpress update-an archive of microarray and highthroughput sequencing-based functional genomics experiments. Nucleic Acids Res. 39, D1002-D1004 (2011).

55. Barrett, T. et al. NCBI GEO: archive for functional genomics data sets-10 years on. Nucleic Acids Res. 39, D1005-D1010 (2011).

56. Dreszer, T.R. et al. The UCSC Genome Browser database: extensions and updates 2011. Nucleic Acids Res. 40, D918-D923 (2012). 